\title{
Case Report Of Solitary Eccrine Spiradenoma Of Eyelid
}

\author{
Sudhir Singh, MS, ${ }^{1}$ Subodh Saraf, MS, ${ }^{2}$ Divyesh Goswami, MD³ and Sumit Singh, DOMS ${ }^{4}$
}

Senior Consultant and Head Of Department, Department of Ophthalmology, JW Global Hospital \& Research Centre, Mount Abu, Rajasthan, India; 2. Ophthalmology Registrar, Global Hospital Institute of Ophthalmology, Sirohi, Rajasthan, India; 3. Consultant Pathologist, JW Global Hospital \& Research Centre, Mount Abu, Rajasthan; 4. Consultant Ophthalmologist, Mathura, Uttar Pradesh, India

\begin{abstract}
Eccrine spiradenoma, a sweat gland tumor, is rarely found in the eyelid. We encountered a 73-year-old man with a nodular growth in his left upper eyelid, which had developed over the last 12 years. He had developed mild pain in the past. Excision was carried out under local anesthesia in a pentagon fashion with clear margins. The histopathology was suggestive for a benign eccrine spiradenoma.
\end{abstract}

\section{Keywords}

Eccrine spiradenoma, eyelid, benign, Sudhir singh

Disclosure: Sudhir Singh, MS, Subodh Saraf, MS, Divyesh Goswami, MD and Sumit Sing DOMS have no conflicts of interest to declare. No funding was received in the publication of this article.

Compliance with Ethical Guidelines: Informed consent was taken from the patient involved in this study.

Received: June 6, 2013 Accepted: August 30, 2013 Citation: touchOPHTHALMOLOGY, online only. DOI: 10.17925/EOR.2015.09.01.80

Correspondence: Sudhir Singh, MS, Senior Consultant and Head of Department, Department of Ophthalmology, JW Global Hospital \& Research Centre, Mount Abu, Rajasthan 307501, India. E: drsudhirsingh@gmail.com

Eccrine spiradenoma are benign tumors of the skin found at the site of eccrine sweat glands. Lesions usually present as solitary, varied-colored, $1 \mathrm{~cm}$ diameter nodules. A review of the literature revealed that only a few cases of eyelid spiradenoma have been reported..$^{1-5}$ In this article, we describe a case of solitary eccrine spiradenoma of the eyelid.

\section{Literature Review}

Eccrine spiradenoma are benign adnexal neoplasms that have been historically designated as a tumor of eccrine differentiation, although current reconsideration indicates an apocrine process. It usually presents on the trunk and extremities as a tender dermal or subcutaneous papule or nodule frequently with a pink or blue hue. The clinical picture is often not distinct and biopsy is required for diagnosis. Eccrine spiradenoma can present in a variety of ways, including as tumors arranged in zosteriform/ dermatomal and/or blaschkoid distributions, often precluding a straightforward diagnosis. Sweat glands present in two ways: eccrine and apocrine. In the eyelid, eccrine sweat glands are present in both the lid margin and in the dermis over the surface of the eyelid. They were first described by Kerstin and Helwig. ${ }^{4}$ Eccrine spiradenoma are solitary, deepseated nodules, covered by normal appearing skin. They are the rarest of eyelid adnexal lesions. ${ }^{6}$ Spiradenoma are usually benign, but proper diagnosis of eccrine spiradenoma is important due to the occurrence of potentially life-threatening malignant transformation.

We reviewed the literature extensively and found that only three cases have been reported so far. All reported cases were females and age ranged from 23 to 60 years (see Table 1).

\section{Case Report}

A 73-year-old man presented with growth on left upper eyelid that had developed gradually over the past 12 years. He had complaint of mild pain in the swelling over the previous few days. On examination the growth was lying on the lateral one-third part of the lid, measured $11 \mathrm{~mm} \times 9 \mathrm{~mm}$, was moderately firm in consistency, and multilobulated (see Figure 1).

The rest of the ophthalmic and systemic examination was insignificant. No lymph nodes were palpable. When considering the long duration of presentation and absence of keratinisation and pigmentation we made a clinical diagnosis of benign sweat gland tumor. With written, informed consent, surgical excision of the growth was planned.

Excision was carried out under local anesthesia in a pentagon fashion with clear margins. The resulting defect involved the lateral onethird part of the lid so it was sutured with silk 4-0 for tarsal plate and 6-0 for skin. The postoperative appearance was of minimal ptosis, which improved with time (see Figure 2). The growth was sent for histopathologic examination.

\section{Gross Examination}

The tumor was a solitary nodule $2.0 \times 1.1 \times 0.8 \mathrm{~cm}$ in size. The consistency was firm and its appearance after cross section was grayish-white.

\section{Microscopic Examination}

Hematoxylin and eosin stain (H\&E) sections revealed epidermis with underlying tumor showing round nodules of basaloid cells. The nodules also revealed some pale cells with vesicular nuclei. Pseudo rosettes with central basement material were present.

\section{Discussion}

There are two types of sweat glands: eccrine and apocrine. In the eyelid, eccrine sweat glands are present in both the lid margin and 
Figure 1: Left Upper Lid Eccrine Spiradenoma

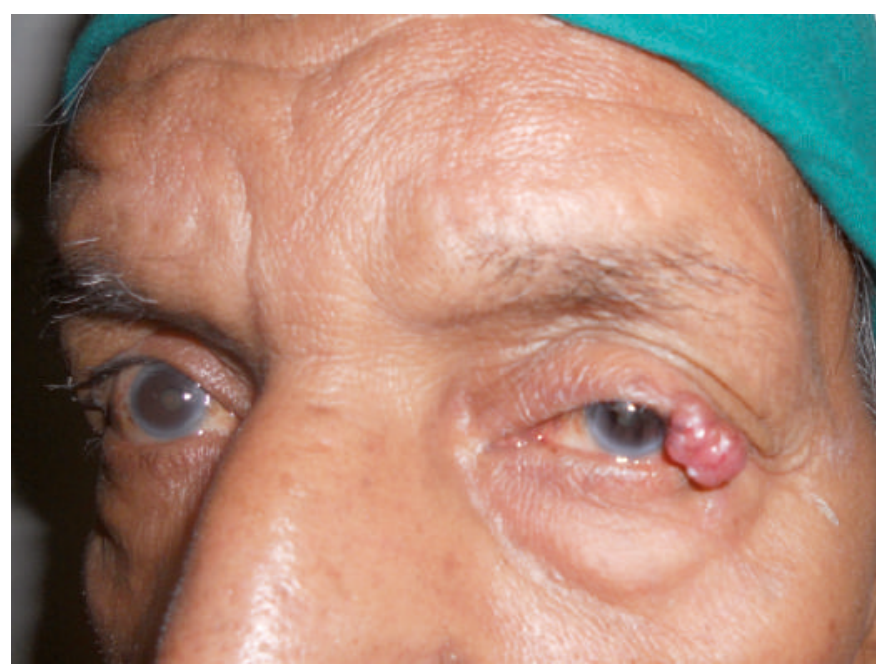

Figure 3: Eccrine Spiradenoma of the Eyelid

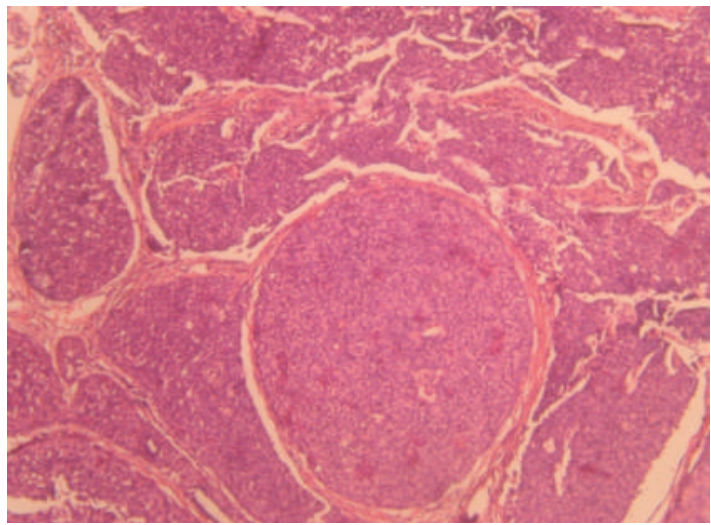

Hematoxylin and eosin (10x) stain section showing round nodules of basaloid cells.

in the dermis over the surface of the eyelid. Eccrine spiradenoma are the rarest of eyelid adnexal lesions. ${ }^{5}$ They are solitary, deep-seated nodules, covered by normal appearing skin. Spiradenoma are usually benign and mostly appear between 15 and 35 years of age. It generally appears between 15 to 35 years of age. No sexual predilection has been reported. About five cases have been reported on eyelid. In India, Ahluwalia et al. reported solitary eyelid spiradenoma in 1986. Noto and co-workers reported linear spiradenoma arising in medial canthus and cheek.? Gupta et al. reported a 23-year-old woman with linear facial spiradenoma and coexistent eyelid spiradenoma. ${ }^{3}$ There have been some reports of malignant transformation of spiradenoma. Histopathologically a connective tissue often surrounds lobulated to multilobulated lesion. The lobules consist of basophilic cells arranged in rosettes. Cells with dark nuclei and larger lighter staining nuclei are present. ${ }^{8}$ Cylindromas should be mentioned as differential diagnosis of spiradenoma. Spiroadenomas can also coexist in the same lesion as cylindromas. In cylindromas, basoloid cells are arranged in 'islands' that fit together like a jigsaw puzzle, rather than in 'rosettes' as in eccrine

\section{Figure 2: Postoperative Appearance after Excision of the Eccrine Spiradenoma}

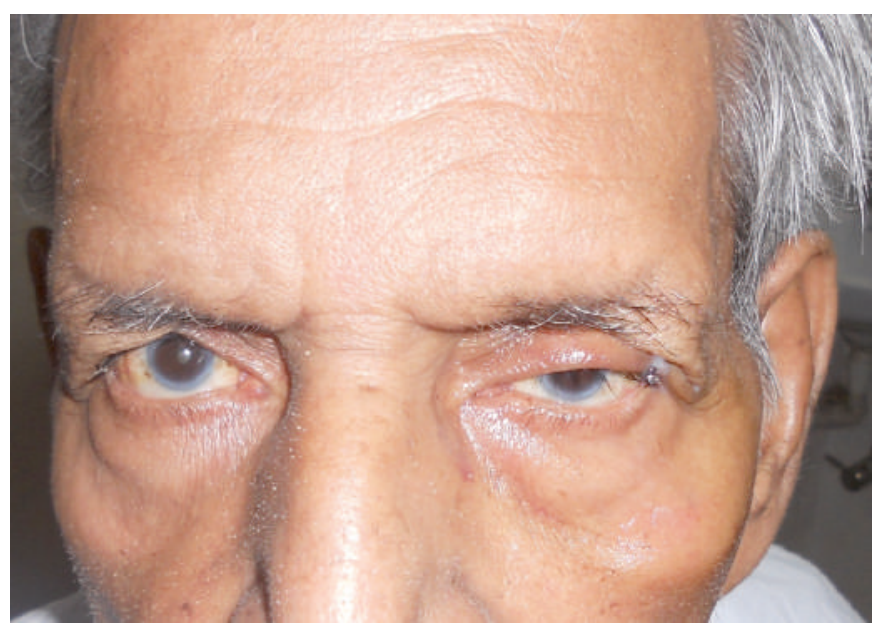

\section{Figure 4: Eccrine Spiradenoma of the Eyelid}

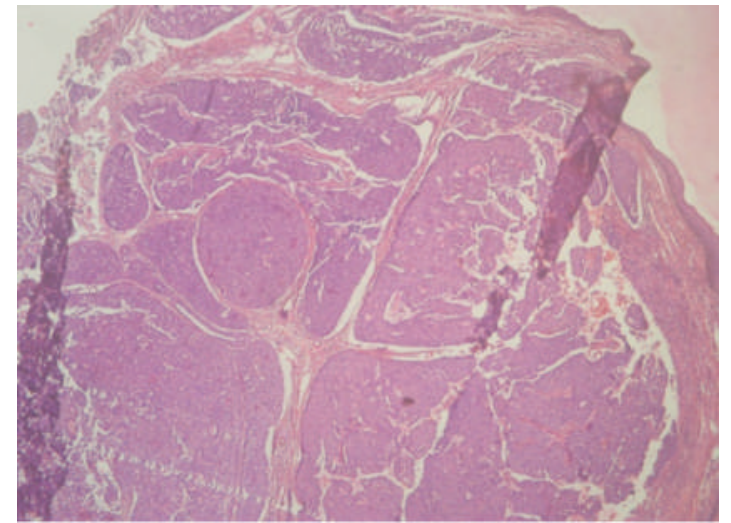

Hematoxylin and eosin (4x) stain section showing sharply delineated, basophilic nodules in the dermis (cannon balls or big blue balls in the dermis).

\section{Table 1: Published Cases of Eccrine Spiradenoma of the Eyelid}

\begin{tabular}{lllll} 
Author & Age/Gender & Location & Size & Number \\
Ahluwalia et al. $^{1}$ & 55/Female & Left upper lid & $24 \times 20 \times 12 \mathrm{~mm} 1$ \\
\hline Amann et al. $^{2}$ & 60/Female & Right upper lid & $10 \times 8 \mathrm{~mm}$ & 1 \\
\hline Gupta et al. $^{3}$ & 23/Female & Left lid & & 1
\end{tabular}

spiradenomas. ${ }^{5}$ The mainstay of treatment is surgical removal. The lesion does not usually recur after excision. Radiation, chemotherapy, and hyperthermic limb perfusion chemotherapy have been carried out in malignant spiradenomas.

In terms of our report, eccrine spiradenomas should be kept in mind when considering the differential diagnosis of eyelid tumors. Among the rare reported cases of eccrine spiradenomas of the eyelid, two have been reported from India. Our case is the third reported case from India.

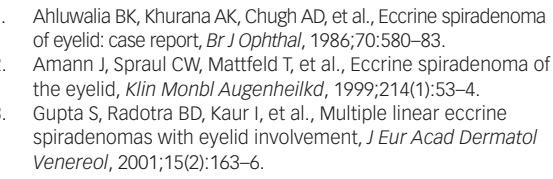
of eyelid: case report, Br J Ophthal, 1986;70:580-83.

Amann J, Spraul CW, Mattfeld T, et al., Eccrine spiradenoma of the eyelid, Klin Monbl Augenheilkd, 1999;214(1):53-4.

Gupta S, Radotra BD, Kaur I, et al., Multiple linear eccrine spiradenomas with eyelid involvement, J Eur Acad Dermatol venereol, 2001;15(2):163-6.

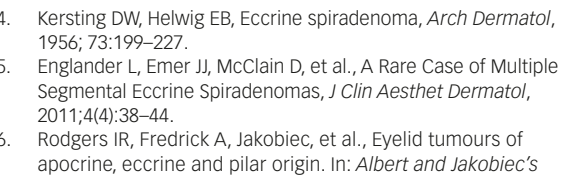

Kersting DW, Helwig EB, Eccrine spiradenoma, Arch Dermatol, 1956; 73:199-227.

Englander L, Emer JJ, McClain D, et al., A Rare Case of Multiple Segmental Eccrine Spiradenomas, I Clin Aesthet Dermatol, 2011;4(4):38-44.

6. Rodgers IR, Fredrick A, Jakobiec, et al., Eyelid tumours of apocrine, eccrine and pilar origin. In: Albert and Jakobiec's

Principles and Practice of Ophthalmology 3rd edition 2008:3:3343.

Noto G, Bongiorno MR, Pravia G, et al., Multiple nevoid spiradenomas, Am J Dermatopathol, 1994;16(3):280-84.

8. Cooper PH, Frierson HF Jr, Morrison AG, Malignant transformation of eccrine spiradenoma, Arch Dermatol, 1985; 121(11):1445-8. 